\title{
Use of Stokes and anti-Stokes Raman scattering for new applications
}

\author{
Thomas H. Kauffmann ${ }^{1,2}$, Ninel Kokanyan ${ }^{1,2}$ and Marc D. Fontana ${ }^{1,2}$ \\ ${ }^{1}$ Université de Lorraine, Laboratoire Matériaux Optiques, Photonique et Systèmes, Metz, F-57070, France. \\ ${ }^{2}$ Laboratoire Matériaux Optiques, Photonique et Systèmes, CentraleSupélec, Université Paris-Saclay, Metz, F-57070, \\ France.
}

\begin{abstract}
We show the interest in recording both Stokes and anti-Stokes Raman spectra in two peculiar applications. In one case the comparison between these two parts of the spectrum, measured at one temperature only, allows to distinguish between first- and second- order phonon bands. In the second example, the difference between Stokes and anti-Stokes spectra provides separation between vibrational and emission lines. We describe the different stages needed in the measurements and data treatment.
\end{abstract}

Keywords : Stokes and anti-Stokes Raman scattering, luminescence, phonon bands, first and second order scattering, population factor.

\section{Introduction}

Raman spectroscopy is a well-known technique that provides chemical and structural information of various compounds, from the measurement of vibrational states of a molecule or compound. ${ }^{[1]}$ It is derived from inelastic scattering of a laser radiation by matter. Raman shifted photons have either lower energy (longer wavelength)- it is the Stokes scattering -or higher energy (shorter wavelength) than the elastic Rayleigh line- it is the anti-Stokes scattering. In each case one vibrational quantum of energy (phonon) is gained or lost, so that the Stokes and anti-Stokes lines are equally shifted from the Rayleigh line. As phonons are bosons, the intensity of Stokes and anti-Stokes lines are proportional to $(n+1)$ or $n$, respectively, where $n$ is the phonon occupation number. Therefore, as the Stokes line is more intense than the anti-Stokes line, only the Stokes spectrum is generally recorded and analyzed in conventional Raman spectroscopy. ${ }^{[2]}$

The most frequent application utilizing both Stokes and anti-Stokes scattering concerns the determination of temperature $T$ from the ratio of intensity of the Stokes and anti-Stokes of the same Raman peak. ${ }^{[3,4]}$ Even if the position and width of a Raman line vary with temperature, ${ }^{[5,6]}$ only the dependence of the Stokes/anti-Stokes intensity ratio can be efficiently exploited to measure in real time and locally the temperature. In particular this tool was used to estimate the temperature generated by the effect of light radiation only. ${ }^{[7]}$ The other use of anti-Stokes signal is the Coherent Anti-Stokes Raman Scattering (CARS) that results from a nonlinear four-wave mixing process. ${ }^{[8]}$ CARS is utilized to enhance the spontaneous Raman signal that is naturally weak.

Generally, the Stokes scattered spectrum is sufficient to give information of the vibrational modes in a molecule or a compound. However, the bare vibrational Raman spectrum that arises from first-order scattering can be partly or fully hidden by the fluorescence ${ }^{[9]}$ and/or the secondorder (or multiple scattering) process. ${ }^{[10]}$ Well established experimental methods can be applied to resolve these difficulties. In the first case the incident laser wavelength can be changed to avoid luminescence since this occurs within an absolute wavelength spectral range while the Raman scattering results into a shift of energy (wavenumber). ${ }^{[1,9]}$ 
In the last case, the study of the thermal behavior of the intensity of the various peaks can be undertaken. Indeed, as the intensity of the second-order scattering varies as $T^{2}$, they can be distinguished from the first-order scattering (the intensity of which varies as $T$ ). ${ }^{[10,11]}$

In the paper we show that it is possible to solve the above mentioned questions by means of an appropriate treatment of both anti-Stokes and Stokes spectra recorded at one temperature solely and for one exciting laser line only. This alternative method requires at first a perfect calibration of the spectrometer and the introduction of notch filters, to reduce the influence of elastic scattering. Thus in principle a fully symmetric anti-Stokes+Stokes spectrum can be achieved within a wide wavenumber range.

\section{Experimental and treatment processes}

\subsection{Basics}

Within the Raman scattering process the interaction of the laser light with the medium implies the conservation of both energy and wave vector. The energy change corresponds to the creation of one phonon (Stokes process) or destruction of one phonon (anti-Stokes process) according to:

$$
\hbar \omega_{s}=\hbar \omega_{i} \mp \hbar \omega_{p}(1)
$$

where $\hbar$ is the reduced Planck constant, $\omega_{i}, \omega_{s}, \omega_{p}$ are the frequency of the incident photon, the scattered photon and the phonon respectively.

The intensity $I_{s}$ of first-order scattering by a phonon or any excitation described by a boson is proportional to: ${ }^{[12]}$

$$
I_{s} \propto I_{i} \chi^{\prime \prime}(\omega) \omega_{s}^{3}\left\{\begin{array}{c}
n(\omega, T)+1 \\
n(\omega, T)
\end{array}\right.
$$

where $I_{i}$ is the intensity of the input laser, $n(\omega, T)$ is the population factor of phonons at thermal equilibrium given by:

$$
n(\omega, T)=\frac{1}{\exp \left(\frac{\hbar \omega}{k_{B} T}\right)-1}
$$

where $k_{B}$ is the Boltzmann factor and $T$ the absolute temperature. $\chi$ ” $(\omega)$ is the line shape response of a harmonic mode. According to Stokes or anti-Stokes scattering, the factor $n+1$ or $n$ is used. The factor $\omega_{s}^{3}$ reflects the conversion of photon in the CCD i.e. via photon counting device. If the detector is based on energy detection, this factor is $\omega_{s}{ }^{4}{ }^{[7,13]}$

Both factors have to be accounted in order to reach the bare spectral response corresponding to $\chi$ ” $(\omega)$. The first one related to population factor is larger than the second one (factor $\omega_{s}^{3}$ or $\omega_{s}{ }^{4}$ ). Therefore the intensity ratio between Stokes and anti-Stokes is generally approximated by $I_{\text {Stokes }} / I_{\text {anti- }}$ Stokes $\sim(n(\omega, T)+1) / n(\omega, T)$ and used to determinate local temperature. ${ }^{[7]}$ It should be mentioned that this ratio for the second-order scattering is much larger since it varies as $((n(\omega, T)+1) / n(\omega, T))^{2}$.

In vibrational spectroscopy, it is frequent to use the wavenumber $\bar{v}=1 / \lambda$ (expressed in $\mathrm{cm}^{-1}$ ) instead of the angular frequency, so that the relative wavenumber $\Delta \bar{v}=1 / \lambda_{i}-1 / \lambda_{s}$ describes the Raman shift with respect of the laser line at $0 \mathrm{~cm}^{-1}$. In this case Equation (1) can be re-written as follows:

$$
\bar{v}_{s}=\bar{v}_{i} \mp \bar{v}_{p}(4)
$$


Note that the upper symbol corresponds to the Stokes scattering in both cases (Equations 1 and 4).

\subsection{Experimental conditions and features}

Raman spectra were recorded using a confocal Raman microscope LabRAM HR Evolution (Horiba) with an exciting line of $532 \mathrm{~nm}$. The laser light was focused through a $50 \times$ objective (Olympus) at a distance above $1 \mathrm{~cm}$ from the sample placed into a HFS temperature stage (Linkam). The Raman system leads to a spectral resolution of $1 \mathrm{~cm}^{-1}$ using a 1800 grooves per millimeter grating and is equipped with an ultra-low frequency (ULF) module. The ULF module consists of three Notch filters in a row rejecting the laser line centered at $0 \mathrm{~cm}^{-1}$ and providing access to antiStokes scattering and wavenumbers down to $\pm 5 \mathrm{~cm}^{-1}$. In the examples illustrating our proposed method, we carried out backscattering Raman measurements in various polarized configurations in order to discriminate different phonon modes according to their symmetry.

\subsection{Procedure and treatments}

To correctly simultaneously use the Stokes and anti-Stokes parts of a Raman spectrum, it is necessary to pay attention to some experimental features and then to apply several treatments. The procedure consisting of applying a series of operations is described below.

At first, the use of several notch filters is needed to reject the main part of elastic scattering. The exploitation of the spectrum in both sides of the Rayleigh line, additionally requires a special attention on the adjustment of the position of zero line. Obviously the spectra are to be calibrated to a well known line (i.e. Si peak located at $520.7 \mathrm{~cm}^{-1}$ ).

Then, we can proceed by successive steps of treatment as follows.

The first stage consists in symmetrizing the Stokes and the anti-Stokes parts with respect to the laser line. For this, the data are interpolated to increase the number of points that defines the spectrum (step of $0.2 \mathrm{~cm}^{-1}$ ). The laser line can be then adjusted more precisely to $0 \mathrm{~cm}^{-1}$ by shifting the spectrum along the wavenumber axis. Moreover, this interpolation leads to have the same step in $\mathrm{cm}^{-1}$ between two consecutive points along the entire spectrum on the Stokes and the anti-Stokes parts.

Second, it is important to account the sources of anti-symmetric background coming from the instrumentation. The background arising from the CCD camera is corrected by a white light spectrum and stored in the software. Then a specific linear baseline correction is applied to reduce the effect of the notch filters. As the Raman intensity is proportional to $\omega_{s}{ }^{3}$, when a CCD is used as detector $^{[7,13]}$ it needs to be corrected by dividing the spectrum by this factor. The next step is to take into account the population factor given by Equation (3). Thus, the intensities from the anti-Stokes part (inferior to $0 \mathrm{~cm}^{-1}$ ) are divided by $n$ and the intensities from the Stokes part (superior to $0 \mathrm{~cm}^{-1}$ ) by $n+1$.

After these corrections, we can proceed to the last step consisting in the difference between the Stokes and the anti-Stokes parts. We therefore obtain the so-called difference spectrum as follows:

$$
I_{\text {Difference }}=\frac{I_{\text {Stokes }}}{(n(\Delta \bar{v}, T)+1) \cdot v_{i} \cdot\left(v_{i}-\Delta \bar{v}\right)^{3}}-\frac{I_{\text {anti-Stokes }}}{n(\Delta \bar{v}, T) \cdot v_{i} \cdot\left(v_{i}+\Delta \bar{v}\right)^{3}}
$$

In these calculations, the population factor is used as follows:

$$
n(\Delta \bar{v}, T)=\frac{1}{\exp \left(\frac{1.439 \times \Delta \bar{v}}{T}\right)-1}
$$

where $\Delta \bar{v}$ is the relative wavenumber in $\mathrm{cm}^{-1}$. 
In our procedure, the difference between the Stokes and anti-Stokes parts is used rather than the ratio since it is more sensitive.

Consistently with Equation (5) if the difference spectrum is zero, this shows without any ambiguity the first-order scattering of the spectrum.

To illustrate the correction procedure we have recorded the Raman spectrum on cystine and we used it as example. As shown in Figure 1, we note the discrepancy between raw data and the spectrum obtained after application of the corrections. In this case we achieve, a nearly perfect and symmetrical spectrum, so that the spectrum difference is almost zero. However, it should be mentioned that due to the laser filters, the signal between -5 and $+5 \mathrm{~cm}^{-1}$ has not to be considered.
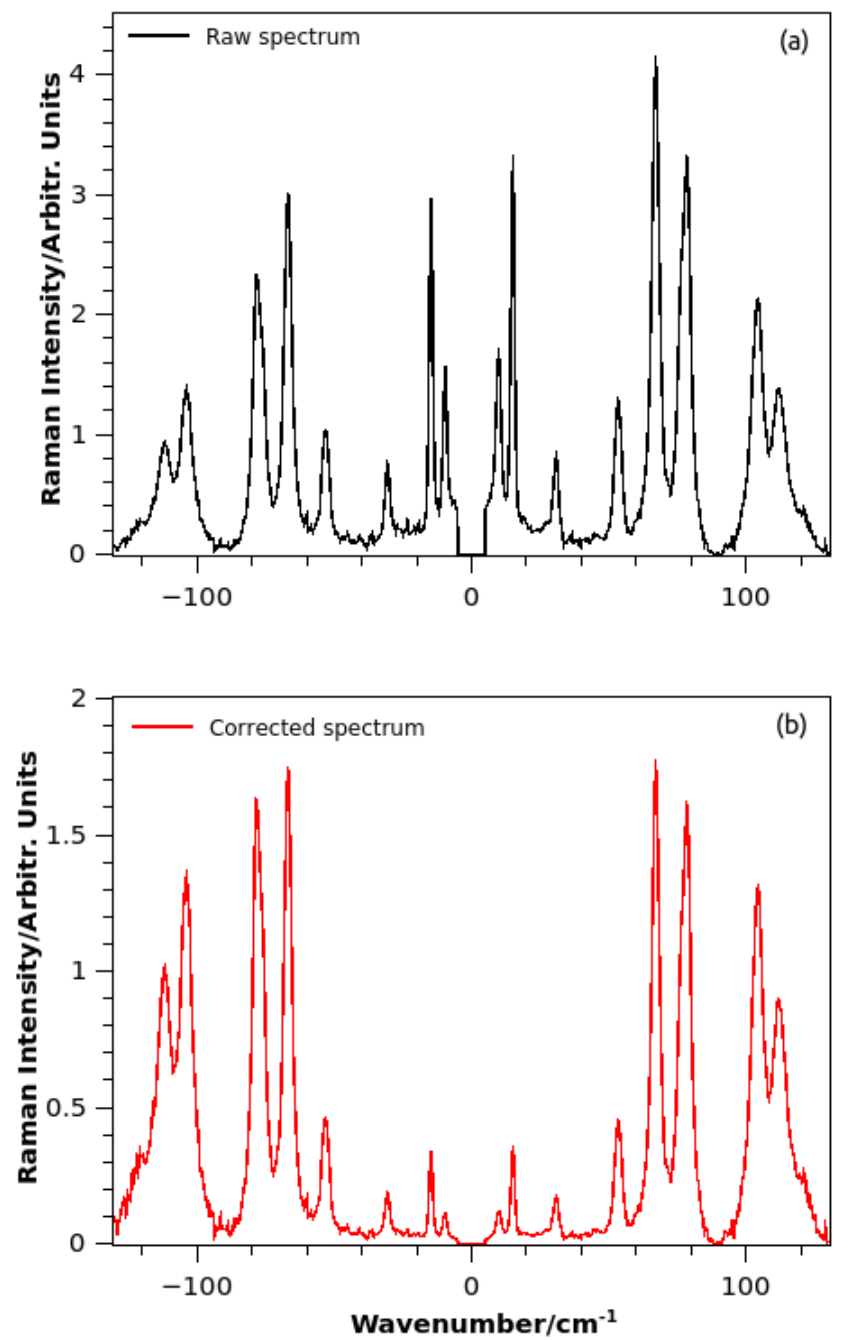

Figure 1. Raman spectrum of cystine (a) before and (b) after the correction procedure.

\section{Results and applications}

\subsection{Second-order scattering and line leakage}

The first example concerns the highlighting of expected first-order lines in a polarized Raman spectrum. Indeed, additional bands can be due either to second-order scattering or to a leakage of modes of a symmetry different from the expected one. These both cases are illustrated by $\mathrm{BaTiO}_{3}$ (BT). BT is a material of perovskite structure, undergoing successive ferroelectric phase transitions on cooling from the paraelectric cubic phase. ${ }^{[14]}$ This compound was widely investigated by Raman spectroscopy in order to study the mechanism of phase transitions. ${ }^{[15,16,17]}$ We show here two different examples on polarized Raman spectra recorded on this material. The use of various 
configurations gives rise, in principle, to a discrimination of phonon modes with different symmetries.

The Raman spectrum carried out at room temperature and reported in Figure 2 presents, in the configuration corresponding to the detection of $\mathrm{E}(\mathrm{TO})$ modes in low-wavenumber range, an intense signal due to the superposition of strong quasi-elastic scattering and low energy phonon. These features were widely studied in the framework of phase transition mechanism. ${ }^{[17,18]}$ Here we pay attention to the broad band and the narrow line which are detected at higher wavenumber. The sharp peak around $300 \mathrm{~cm}^{-1}$ is without any ambiguity attributed to first-order scattering due to optical E phonon. By contrast, the broad bands are the object of controversies about their origin: second-order scattering or disorder-induced first-order scattering. ${ }^{[15]}$ We therefore applied the above mentioned procedure, in order to discriminate between first and second order origin of the band around 270 $\mathrm{cm}^{-1}$. The "difference spectrum" was obtained nearly to 0 . This means that both lines are attributed to first-order phonons. In fact, narrow peaks are expected only in the configuration $\mathrm{Y}(\mathrm{XZ}) \mathrm{Y}$, while wide bands result from a leakage of first-order phonons with another symmetry ( $\mathrm{A}_{1}$ modes) owing to a small misorientation of the sample versus polarizers (or imperfection of polarizers). ${ }^{[19]}$
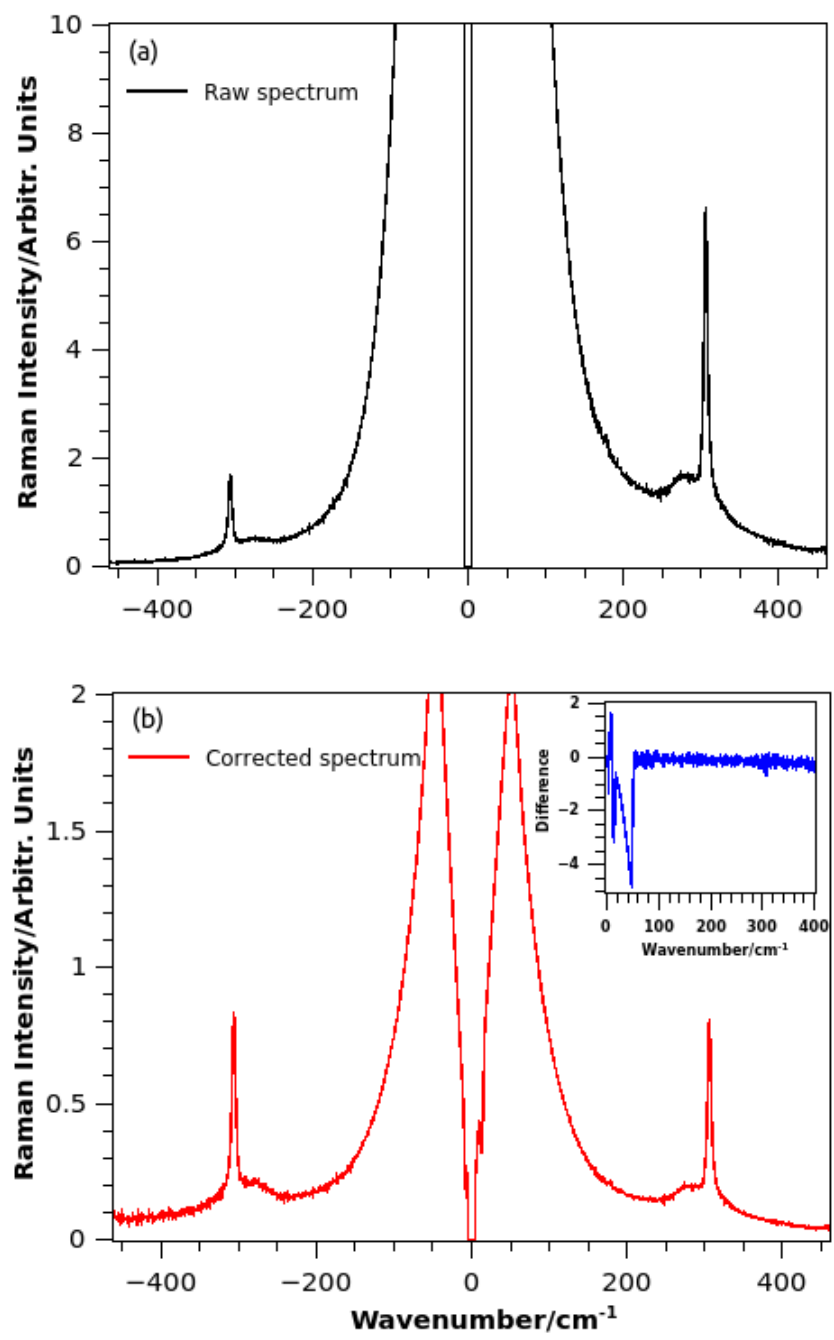

Figure 2. Spectra recorded on BT in the $\mathrm{Y}(\mathrm{XZ}) \mathrm{Y}$ configuration at room temperature. (a) raw data; (b) spectrum after applying corrections; (insert) difference spectrum. The "zero" difference above $50 \mathrm{~cm}^{-1}$ between Stokes and anti-Stokes spectra reported in the insert proves the first-order scattering origin of the narrow and broad peaks. The signal below $50 \mathrm{~cm}^{-1}$ is due to the filter and quasi-elastic scattering which is not fully symmetric. 
The spectrum shown in Figure 3 and recorded at $180^{\circ} \mathrm{C}$ in the cubic phase of BT displays only wide bands. In principle, only second-order scattering is expected in the centrosymmetric structure. Nevertheless, several authors invoked the possibility of first-order induced by Ti-disorder to explain the large signal. ${ }^{[20,21,22]}$ According to our approach, a difference spectrum should be vanished in this case. Our calculations give rise to a non-zero "difference spectrum" (see the insert Figure $3 b$ ). This clearly discards the sole first-order origin of the detected broad bands and proves by contrast that they mainly arise from second-order scattering. However the possibility of weak first-order scattering hidden by strong and broad bands cannot be excluded within our procedure.

The separation between first- and second- order scattering is a problem that is difficult to be solved. It generally requires to exploit the temperature dependence of the spectrum since the population factor is strongly different for both processes. ${ }^{[19]}$

Here the advantage of our procedure is that the measurements are needed at one temperature only.
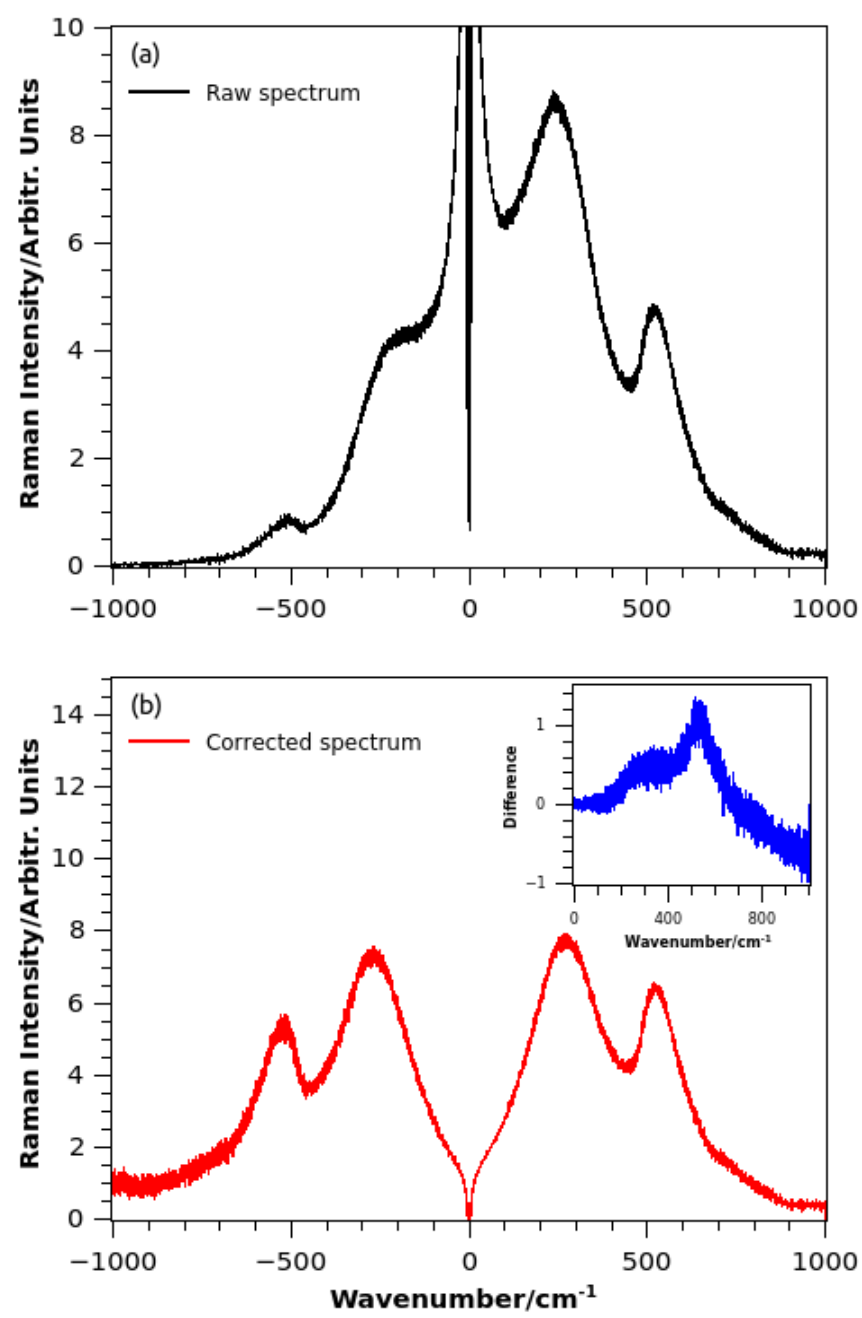

Figure 3. Spectra recorded on $\mathrm{BT}$ in the $\mathrm{Z}(\mathrm{XX}) \mathrm{Z}$ configuration at $180^{\circ} \mathrm{C}$ (cubic phase). The difference between Stokes and anti-Stokes spectra reported in the insert evidences the second-order origin of the scattering.

\subsection{Superposition of emission and vibrational states in the spectrum}

The second application concerns the simultaneous observation of Raman bands and emission lines, within the same range of the recorded spectrum in a crystal of $\mathrm{LiNbO}_{3}$ doped with holmium. Lithium niobate (LN) is a well-known material thanks to its outstanding properties ${ }^{[23,24,25]}$ and 
potential applications in piezoelectricity, acoustics, ${ }^{[26,27]}$ wave guiding ${ }^{[28]}$ and nonlinear-optics ${ }^{[29]}$ It is a versatile material, the properties of which can be matched according to the nature and concentration of doping. ${ }^{[30,31]}$ Thus, doping these crystals with rare earth ions attracts attention for laser applications, and therefore characterization of emission spectra is required in this case. Doping with holmium presents a special interest for up-conversion emission in green and red. ${ }^{[32,33]}$ We thus carried out polarized Raman experiments on $\mathrm{Ho}^{3+}$-doped LN single crystals.
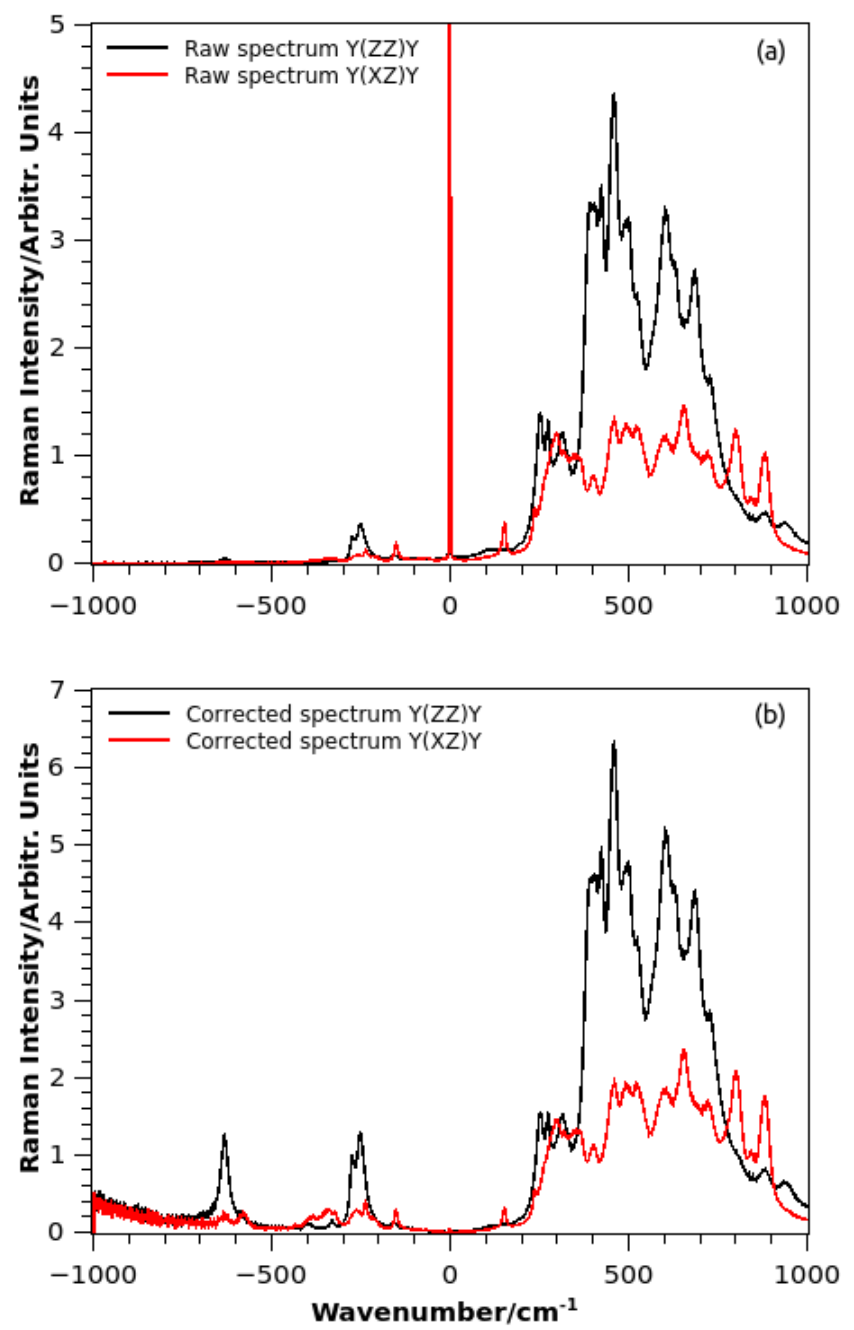

Figure 4. Spectra recorded on $1 \%$ mol $\mathrm{Ho}^{3+}$ doped $\mathrm{LN}$ in $\mathrm{Y}(\mathrm{ZZ}) \mathrm{Y}$ and $\mathrm{Y}(\mathrm{XZ}) \mathrm{Y}$ configurations. (a) raw data; (b) spectra after corrections.

The spectra were recorded in the configurations $\mathrm{Y}(\mathrm{ZZ}) \mathrm{Y}$ and $\mathrm{Y}(\mathrm{XZ}) \mathrm{Y}$ corresponding to $\mathrm{A}_{1}(\mathrm{TO})$ and E(TO) phonon modes respectively. Figure 4a shows the Raman spectrum recorded on LN: 1\% mol Ho with $532 \mathrm{~nm}$ exciting laser line. The spectrum exhibits a Stokes part totally different from the anti-Stokes part. Such a large difference is rather unusual, and can be explained by the detection in the Stokes part, of emission peaks besides Raman lines. This is confirmed if the excitation wavelength is changed to $785 \mathrm{~nm}$, because in this case, the Raman spectrum is only detected with symmetrical Stokes and anti-Stokes positions. Furthermore the anti-Stokes spectrum in Figure 4 exhibits the expected first-order lines in agreement with earlier Raman data. ${ }^{[34]}$

The Stokes Raman spectrum of Figure 4 is difficult to analyze due to the superposition of the vibration and emission bands. It should be underlined that here the emission lines are as narrow as vibrational lines, rendering impossible the discrimination between both phenomena. We therefore applied the procedure described above. Since the luminescence only causes a Stokes shift only, the 
less intense anti-Stokes part reported in Figure 5a could be used to study the sole vibrational modes. The "difference spectrum" plotted in Figure 5b shows that, as expected, the Raman peaks completely disappear and reveals only the emission spectrum in both configurations. We thus achieved a separate detection of both vibrational and emission spectra as illustrated in Figure 5.

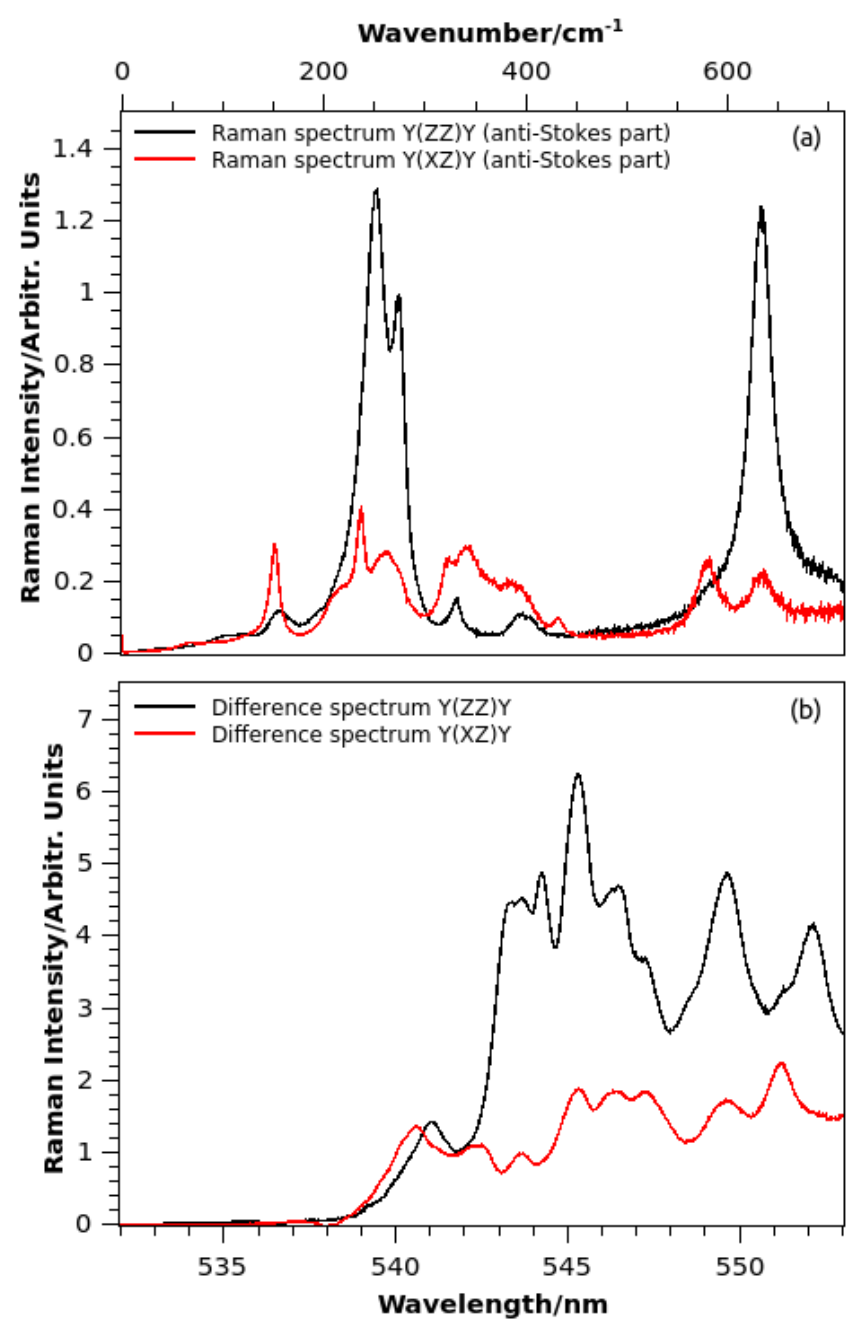

Figure 5. Spectra of $1 \%$ mol $\mathrm{Ho}^{3+}$ doped $\mathrm{LN}$ in $\mathrm{Y}(\mathrm{ZZ}) \mathrm{Y}$ and $\mathrm{Y}(\mathrm{XZ}) \mathrm{Y}$ configurations. (a) anti-Stokes part after corrections; (b) difference spectra providing $\pi$ and $\sigma$ emission corresponding to $\mathrm{Y}(\mathrm{ZZ}) \mathrm{Y}$ and $\mathrm{Y}(\mathrm{XZ}) \mathrm{Y}$ configurations respectively.

Thus, reconstructed emission and vibrational spectra are found in fairly good agreement with direct and separate luminescence and Raman reported in the literature. ${ }^{[33,35,36]}$

\section{Discussion and conclusion}

A methodology of treatment of both Stokes and anti-Stokes spectra is proposed in order to provide a non-classical exploitation of Raman data. We first underline the corrections afforded by BoseEinstein population factor and the scattered intensity dependence on wavelength. Then we apply our method to two different experimental cases.

In the first example on Raman spectra of $\mathrm{BaTiO}_{3}$ crystal, our suggested procedure allows to discern between the first- or second- order origin of broad bands, from measurements at one temperature only. The multi-scattering processes arise from various combinations of acoustic and optical phonons, and their positions can be calculated by ab initio approach. ${ }^{[1]}$ Nevertheless it is generally difficult to discriminate in an experimental spectrum between lines arising from different 
processes. However, since the population factor is different, the analysis of thermal behavior of intensity of the lines should provide separation. In fact, this method is not reliable since the temperature dependence of the Raman spectrum is function of other parameters such as the sample optical absorbance. ${ }^{[37]}$ A more suitable method consists into fitting the Raman spectrum assuming that the narrow lines are due to first-order phonons and the broad bands arise from multi-phonon process. ${ }^{[19]}$ Our present method is more direct and does not require the study of the temperature dependence of the Raman spectrum.

The second example concerns $\mathrm{Ho}^{3+}$ doped LN crystals with a luminescence superimposed on the Raman spectrum. Our method provides a possibility to separate the emission spectrum which corresponds to the "difference spectrum" between Raman Stokes and anti-Stokes spectra.

Several techniques exist to overcome the fluorescence problem which partly or totally overwhelms the Raman spectrum.

Mosier-Boss et al. ${ }^{[38]}$ and Zhao et al. ${ }^{[39]}$ demonstrated the use of shifted-spectra difference technique for rejecting fluorescence. The technique consists of subtracting two slightly shifted Raman spectra obtained by moving the spectrometer grating ${ }^{[38]}$ or by using two different laser lines. ${ }^{[39]}$ As the fluorescence does not change with the excitation wavelength, the difference Raman spectrum allows to rebuilt properly the pure Raman spectrum free of fluorescence common to both spectra by different signal treatments (edge detection, Fourier transformation, deconvolution algorithms).

All these methods to recover Raman signal, require large instrumentation, several laser sources and sophisticated treatment. They are therefore costly and time consuming. By contrast, our technique is rather rapid and does not request additional set up. In addition, if needed, both emission and vibrational spectra can be separately obtained.

\section{References}

[1] M. Schmitt, J. Popp, J. Raman Spectrosc. 2006 ; 37, 20.

[2] K. Nakamoto, Infrared and Raman spectra of inorganic and coordination compounds, Parts $A$ and $B$, Wiley, 2009.

[3] M. Malyj, J.E. Griffiths, Appl. Spectrosc. 1983 ; 37 , 315.

[4] S.D. McGrane, D.S. Moore, P.M. Goodwin, D.M. Dattelbaum, Appl. Spectrosc. 2014 ; 68 , 1279.

[5] Ph. Colomban, G. Lucazeau, J. Chem. Pys. 1980 ; 72 , 1213.

[6] G. Lucazeau, J. Raman Spectrosc. 2003 ; $34,478$.

[7] J.J. Gallardo, J. Navas, D. Zorrilla, R. Alcantara, D. Valor, C. Fernandez-Lorenzo, J. MartinCalleja, Appl. Spectrosc. 2016 ; 70, 1128.

[8] R. Begley, A. Harvey, R. Byer, Appl. Phys. Lett. 1974 ; 25 , 387.

[9] T. Rojalin, L. Kurki, T. Laaksonen, T. Viitala, J. Kostamovaara, K.C. Gordon, L. Galvis, S. Wachsmann-Hogiu, C.J. Strachan, M. Yliperttula, Anal. Bioanal. Chem. 2016 ; 408 , 761.

[10] P. Brüesch. Springer Series in Solid-State Sciences. 1986 ; 65.

[11] A.D. Bruce, R.A. Cowley, J. Phys. C: Solid State Phys. 1972 ; 5 , 595.

[12] W. Hayes, R. Loudon, in Scattering of Light by Crystals. Wiley-lnterscience, New York, 1978.

[13] B. Schrader, D.S. Moore. Pure \& Appl. Chem. $1997 ; 69,1451$.

[14] M.E. Lines and A.M. Glass, in Principles and Applications in Ferroelectrics and Related Materials. Clarendon Press, Oxford. 1977.

[15] A. Scalabrin, A.S. Chaves, D.S. Shim, S.P.S. Porto, Phys. Status Solidi B. 1977 ; 79 , 731.

[16] M.D. Fontana, K. Laabidi, B. Jannot, J. Phys.: Condens. Matter. 1994 ; 6, 8923. 
[17] J. Hlinka, T. Ostapchuk, D. Nuzhnyy, J. Petzelt, P. Kuzel, C. Kadlec, P. Vanek, I. Ponomareva, L. Bellaiche, Phys. Rev. Lett. 2008 ; 101 , 167402.

[18] K. Laabidi, M.D. Fontana, M. Maglione, B. Jannot, K.A. Muller, Euro. Phys. Lett. 1994 ; 26 , 309.

[19] I. Bejaoui Ouni, D. Chapron, H. Aroui, M.D. Fontana, Appl. Phys. A: Mater. Sci. Process. 2016 ; 122,480 .

[20] M.P. Fontana, M. Lambert, Solid State Commun. $1972 ; 10,1$.

[21] A.M. Quittet, M. Lambert, Solid State Commun. 1973 ; 12 , 1053.

[22] M. DiDomenico Jr., S.H. Wemple, S.P.S. Porto, R.P. Baumann, Phys. Rev. 1968 ; 174 , 522.

[23] K.K. Wong. in Properties of lithium niobate, INSPEC-The Institution of Electrical Engineers, London, U.K. 2002.

[24] T. Volk, M. Wöhlecke, Springer Series in Material Science. $2008 ; 115$.

[25] L. Arizmendi, Phys. Status Solidi A. 2004 ; 201, 253.

[26] J. Kushibiki, Y. Ohashi, Y. Ono, IEEE Transactions on Ultrasonics, Ferroelectrics, and Frequency Control. 2000 ; 47, 1068.

[27] T. Aubert, N. Kokanyan, H. Alhousseini, A. Taguett, F. Bartoli, J. Streque, H. M'Jahed, P. Boulet, O. Elmazria, SENSORS, 2017 IEEE. 2017 ; 1-3.

[28] M. Bazzan, C. Sada, Appl. Phys. Rev. 2015 ; 2 , 040603.

[29] P. Ferraro, S. Grilli, P. De Natale, Springer Series in Materials Science (Springer). 2008 ; 91.

[30] M. Bazzan, M.D. Fontana, Appl. Phys. Rev. 2015 ; 2 , 040501.

[31] E.P. Kokanyan, L. Razzari, I. Cristiani, V. Degiorgio and J.B. Gruber, Appl. Phys. Lett. 2004 ; 84,1880 .

[32] E. Barnes, N.H. O’Connell, N.R. Balli, M. Pokhrel, A. Movsesyan, E. Kokanyan, D. Sardar, Opt. Express. $2014 ; 22,26222$.

[33] A. Lorenzo, L.E. Bausa, J.A. Sanz Garcia, J. Garcia Solé, J. Phys.: Condens. Matter. 1996 ; 8 , 5781.

[34] M.D. Fontana, P. Bourson, Appl. Phys. Rev. $2015 ; 2$, 040602.

[35] A. Ridah, P. Bourson, M.D. Fontana, G. Malovichko, J. Phys.: Condens. Matter. 1997 ; 9 , 9687.

[36] N. Kokanyan, D. Chapron, M.D. Fontana, Appl. Phys. A: Mater. Sci. Process. 2014 ; 117 , 1147.

[37] S. Xie, E. Iglesia, A.T. Bell, J. Phys. Chem. B. $2001 ; 105,5144$.

[38] P.A. Mosier-Boss, S.H. Lieberman, R. Newbery, Appl. Spectrosc. 1995 ; 49 , 630.

[39] J. Zhao, M.M. Carrabba, F.S. Allen, Appl. Spectrosc. 2002 ; 56, 834. 